\title{
Dynamic molecular ordering in multiphasic nanoconfined ionogels detected with time-resolved diffusion NMR
}

\section{Marina Karagianni}

National Centre of Scientific Research Demokritos https://orcid.org/0000-0002-7810-0947

Lydia Gkoura

New York University https://orcid.org/0000-0001-5298-6498

Nikolaos Tsolakis

NCSR Demokritos

Georgios Romanos

NCSR Demokritos

Savvas Orfanidis

NCSR Demokritos

Nikolaos Panopoulos

NCSR Demokritos

Saeed Alhassan

Khalifa University

Aris Chatzichristos

Khalifa University

Jamal Hassan

Khalifa University

Michael Fardis

NCSR Demokritos

Georgios Papavassiliou ( $\nabla$ g.papavassiliou@inn.demokritos.gr)

NCSR Demokritos https://orcid.org/0000-0002-4119-3959

\section{Letter}

Keywords:

Posted Date: January 7th, 2022

DOI: https://doi.org/10.21203/rs.3.rs-1052911/v1 
License: (c) (i) This work is licensed under a Creative Commons Attribution 4.0 International License. Read Full License 


\section{Dynamic molecular ordering in multiphasic nanoconfined ionogels detected with time-resolved diffusion NMR}

M. Karagianni ${ }^{1}$, L. Gkoura ${ }^{2}$, N. Tsolakis ${ }^{1}$, G. Romanos ${ }^{1}$, S. Orfanidis ${ }^{1}$, N. Panopoulos ${ }^{1}$, S. Alhassan $^{3}$, A. Chatzichristos ${ }^{4}$, J. Hassan $^{4 *}$, M. Fardis ${ }^{1 *}$, and G. Papavassiliou ${ }^{1 *}$

${ }^{1}$ Institute of Nanoscience \& Nanotechnology, NCSR Demokritos, 15310 Agia Paraskevi, Attiki, Greece

${ }^{2}$ Division of Science, New York University Abu Dhabi, Abu Dhabi, UAE

${ }^{3}$ Department of Chemical Engineering, Khalifa University of Science and Technology, 127788, Abu Dhabi, UAE

${ }^{4}$ Department of Physics, Khalifa University of Science and Technology, 127788, Abu Dhabi, UAE

*corresponding authors: jamal.hassan@ku.ac.ae,m.fardis@inn.demokritos.gr, g.papavassiliou@inn.demokritos.gr

Molecular motion in nanosized channels can be highly complicated. For example, water molecules in hydrophobic nanopores move rapidly and coherently in a chain, following the so-called single file motion ${ }^{1}$. Surprisingly, fast molecular motion is also observed in viscous charged fluids, such as room temperature ionic liquids (RTILs) confined in a nanoporous carbon or silica matrix ${ }^{2-6}$. The microscopic mechanism of this intriguing effect is still unclear. Here, by combining NMR diffusion experiments in different relaxation windows with ab-initio molecular dynamics simulations, we show that the imidazolium-based RTIL $[\mathrm{BMIM}]^{+}[\mathrm{TCM}]^{-}$, entrapped in the MCM-41 silica nanopores, exhibits a complex dynamic molecular ordering (DMO); adsorbed RTIL molecules near the pore walls orient almost vertically to the walls, while at the center of the pores anion-cation pairs diffuse collectively in a single file (SFD). Enlightening this extraordinary effect is of primary importance in designing RTIL-based composite materials with tuned electrochemical properties. 
RTILs are organic salts with low melting points, usually consisting of large asymmetric organic cations, such as imidazolium and pyridinium, quaternary ammonium, or phosphonium, paired with inorganic or organic anions exhibiting a varying degree of complexity ${ }^{7}$. In recent years, they have attracted much attention due to their unique and tunable properties, which render them promising alternatives for a broad range of applications in lithium-ion batteries, supercapacitors, solar cells, and gas absorption ${ }^{8-10}$. Similar to other liquids, spatial restriction and low dimensionality significantly affect the RTILs properties; they alter phase transition properties $^{11}$, wetting, layering near-surface walls $^{7}$, and ionic mobility ${ }^{2-6}$. Notably, both experiments and theory show that when the pore-sizes of the host material approach the size of the RTIL molecules, their transport properties are sufficiently enhanced ${ }^{2-6}$. However, the molecular mechanism underlying this kind of fast self-diffusion is still obscure.

One of the most common methods to study fluid diffusion in porous systems is the pulsed-field gradient (PFG) $\mathrm{NMR}^{12}$. An alternative method is to use the strong stray field gradient (SFG) of a superconductive magnet instead of the $\mathrm{PFG}^{13-15}$. For unrestricted 3D diffusion, by applying a Hahn echo $\pi / 2-\tau-\pi$ pulse sequence the nuclear spin echo decays according to formula ${ }^{13,14} h(\tau, G)=h(0) \exp \left(-\frac{2 \tau}{T_{2}}\right) \exp \left(-\frac{2}{3} \gamma^{2} G^{2} D \tau^{3}\right)$ (1), where $\gamma$ is the nuclear gyromagnetic ratio, $\mathrm{G}$ is the $\mathrm{SFG}, \mathrm{T}_{2}$ the spin-spin relaxation time, and $\mathrm{D}$ is the selfdiffusion coefficient. The mean square displacement along the magnetic field gradient is given by the relation ${ }^{16}\left\langle\mathrm{z}^{2}\right\rangle=2 \mathrm{D} \tau$. However, in the case of diffusion in 1D narrow channels, such as CNTs, biological porins ${ }^{17}$, or cylindrical silica nanopores ${ }^{18},\left\langle\mathrm{z}^{2}\right\rangle=2 \mathrm{a} \tau^{\mathrm{K}}$, and the NMR signal attenuation in the SFG is given by relation ${ }^{19-21} h(\tau, G, \kappa)=h(0) \sqrt{\frac{\pi}{8 a \alpha(\tau, G, \kappa)}} \operatorname{erf}[\sqrt{2 a \alpha(\tau, G, \kappa)}]$ (2), where $\alpha(\tau, G, \kappa)=\frac{\left(2^{\kappa}-2\right)}{(\kappa+1)(\kappa+2)} \gamma^{2} G^{2} \tau^{\kappa+2}$, a is the mobility factor, i.e. the $1 \mathrm{D}$ analog of the self-diffusion coefficient $D$, and $\operatorname{erf}[\mathrm{x}]$ is the error function. In the extreme case of ultra-narrow 
pore-channels comparable to the size of the diffusing molecules, the so-called single file diffusion $(\mathrm{SFD})$ takes place ${ }^{16,22,23}$, with $\kappa=0.5$.

The presence of SFD has been detected with both PFG and SFG methods, by varying the magnetic field gradient amplitude and recording the spin-echo decay (SED) signal. In this way, SFD of water in single-wall carbon nanotubes $(\mathrm{CNTs})^{16}, \mathrm{CF}_{4}$ in $\mathrm{AlPO}_{4}-5$ zeolites ${ }^{24}$, as well as of Xenon in dipeptides ${ }^{25}$ was detected. In addition, the anomalous diffusion of ${ }^{3} \mathrm{He}$ in solid hydrogen at $1.4 \mathrm{~K}$ was excellently studied $^{21}$. However, in the case of complex diffusion processes in multiphasic systems, such as transcellular ion diffusion, or ionic motion in ionogels, anomalous diffusion is masked by the conventional diffusion processes.

In this study we have focused on the 1-butyl-3-methyl imidazolium tricyanomethanide $\left.{ }_{[\mathrm{BMIM}}\right]^{+}[\mathrm{TCM}]^{-} / \mathrm{MCM}-41(\mathrm{SBA}-15)$ ionogels. According to the nitrogen porosimetry in Fig. 1a, the mean pore-sizes of MCM-41 and SBA-15 are $\sim 4.09 \mathrm{~nm}$ and $\sim 7.31 \mathrm{~nm}$, respectively, while the size of the $[\mathrm{BMIM}]^{+}$cation is $\sim 0.81 \mathrm{~nm}$. Our DFT calculations (details are provided in the Supplementary Information) show that upon entering into the pores, a layer of $[\mathrm{BMIM}]^{+}[\mathrm{TCM}]^{-}$ molecules adsorbed on the silica walls is formed with the $[\mathrm{BMIM}]^{+}$cations oriented almost vertically to the walls, as demonstrated in the two upper panels of Fig. 1a. Consequently, the space for the diffusion of free RTIL ions in MCM-41 is limited, while inside the pores of SBA15 there is more space for free-tumbling RTIL ions.

In order to resolve adsorbed and "mobile" $[\mathrm{BMIM}]^{+}$cations, ${ }^{1} \mathrm{H}$ NMR Carr-PurcellMeiboom-Gill (CPMG) SED measurements were performed at various temperatures, using fully dehydrated samples. Experiments were carried out at Larmor frequency of $100 \mathrm{MHz}$ in a SFG of $\mathrm{G}=34.7 \mathrm{~T} / \mathrm{m}$. In a CPMG experiment, the SED curve is acquired with the $\pi / 2-\tau-\pi-(2 \tau-\pi)_{\mathrm{n}}$ pulse sequence, decaying exponentially with an effective $\mathrm{T}_{2}^{\prime}$, which includes diffusion, as illustrated in Supplementary equation (3) and Supplementary Figs. 3b-3d. Since $\mathrm{T}_{2}^{\prime}$ of adsorbed molecules in a 
porous system are sufficiently shorter than $\mathrm{T}_{2}^{\prime}$ of free-tumbling bulk-like molecules ${ }^{26}$, NMR signals from adsorbed and free tumbling RTIL ions may in principle be resolved by inverting the experimental CPMG SED curves. For this, a Tikhonov regularization algorithm $^{27,28}$ was implemented, as explained in the Supplementary Information text.

Figs. $1 \mathrm{~b}$ and $1 \mathrm{~d}$ show CPMG SEDs versus time of $[\mathrm{BMIM}]^{+}[\mathrm{TCM}]^{-i n t r u d e d}$ into MCM-41 (blue lines) and SBA-15 (red lines) at RT and 400K, whereas insets show the relevant $\mathrm{T}_{2}^{\prime}$ distributions. In both samples at RT two $\mathrm{T}_{2}^{\prime}$ components are resolved, assigned to the adsorbed (low- $\mathrm{T}_{2}^{\prime}$ ) and free tumbling (high-T ${ }_{2}^{\prime}$ ) RTIL cations, while at $400 \mathrm{~K}$ both samples acquire the same single peaked $\mathrm{T}_{2}^{\prime}$ distribution. This is due to the detachment of the adsorbed $[\mathrm{BMIM}]^{+}$cations at elevated temperatures, as discussed below. Contrary to the CPMGs, the Hahn SEDs are markedly different in the two samples, as shown in Fig. 1c. In bulk RTIL (empty circles), the SED follows unrestricted diffusion, described by equation (1). On the other hand in both MCM41 and SBA-15, Hahn SEDs diverge from the exponential $\tau^{3}$ decay, due to strong intermixing of various diffusing processes. Especially, in the case of MCM-41 a tail is observed at long times, which is a characteristic feature of anomalous diffusion ${ }^{19-21}$. 

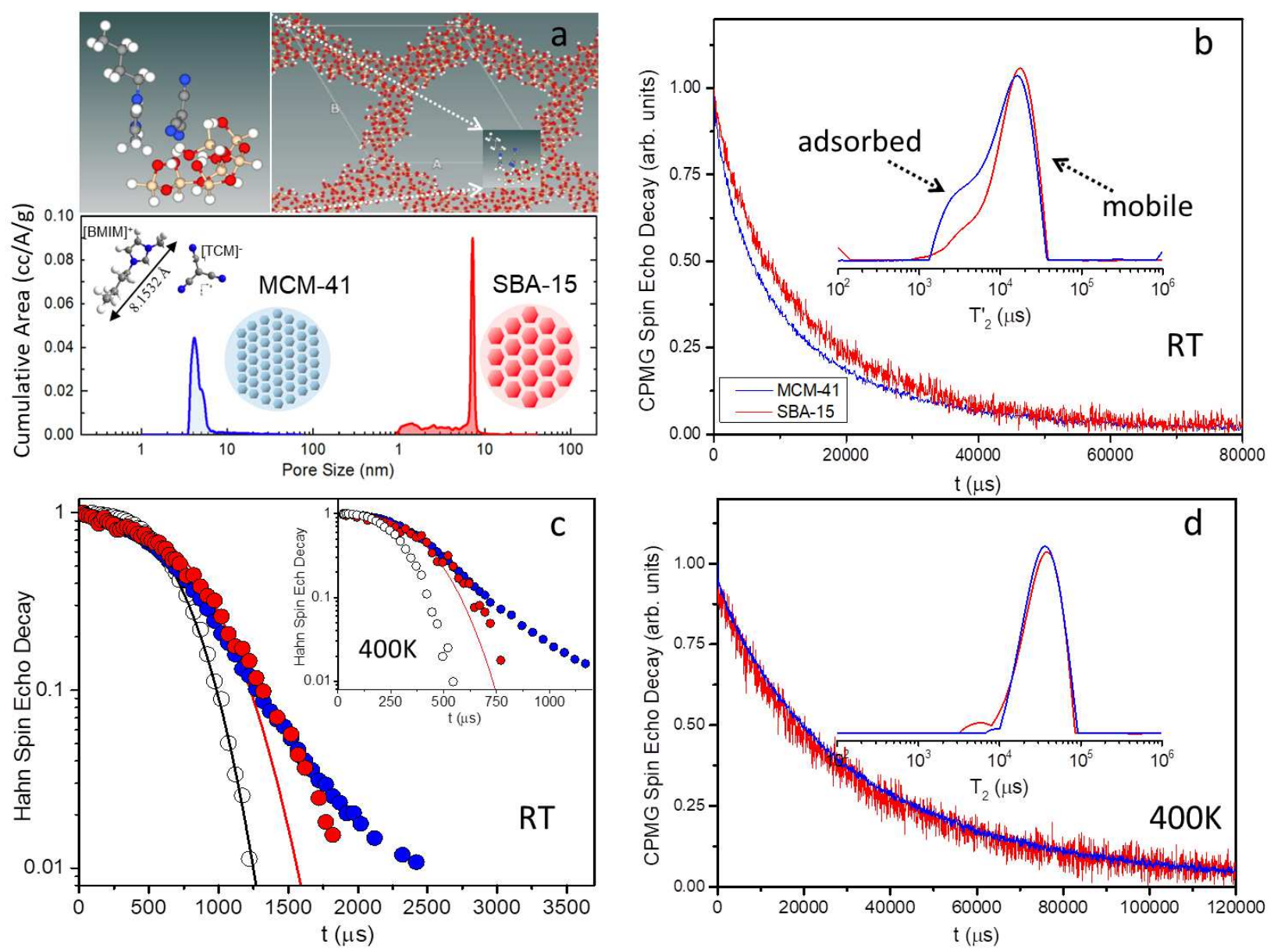

Figure 1 | Adsorbed and mobile $[\mathrm{BMIM}]^{+}$cations resolved with ${ }^{1} \mathrm{H}$ NMR $\mathrm{T}_{2}^{\prime}$ distribution.

a, DFT relaxed $[\mathrm{BMIM}]^{+}[\mathrm{TCM}]^{-}$adsorbed on a protonated $\left[\mathrm{Si}_{6} \mathrm{O}_{12}\right]$ cluster (blue: nitrogen, grey: carbon, white: hydrogen, yellow: silicon, red: oxygen). The upper right panel is a schematic representation of the RTIL's ions adsorbed on the wall of MCM-41. The lower panel shows the liquid nitrogen porosimetry curves of MCM-41 (blue) and SBA-15 (red). A DFT relaxed $[\mathrm{BMIM}]+[\mathrm{TCM}]-$ ion pair is also presented. b, CPMG SEDs at RT of $[\mathrm{BMIM}]^{+}[\mathrm{TCM}]^{-}$ entrapped into MCM-41 (blue line) and SBA-15 (red line). The inset shows the $\mathrm{T}_{2}^{\prime}$ distribution by inverting the CPMG SEDs. c, The Hahn SEDs of $[\mathrm{BMIM}]^{+}[\mathrm{TCM}]^{-}$in bulk form (white circles), as well as inside MCM-41 (blue circles) and SBA-15 (red circles). Solid lines are fits to equation (1). The inset shows the relevant Hahn SEDs at 400K. d, CPMG SEDs at $400 \mathrm{~K}$ of $[\mathrm{BMIM}]^{+}[\mathrm{TCM}]^{-}$entrapped into MCM-41 (blue line) and SBA-15 (red line). The inset shows the 
$\mathrm{T}_{2}^{\prime}$ distribution by inverting the CPMGs.

To acquaint with these intriguing results, we have performed a series of CPMG experiments by varying at each step the time interval $\tau$ between the $\pi / 2$ and first $\pi$ pulse, as explained in Supplementary Fig. 4. In this way, the diffusion footage is encoded in successive CPMGs. Subsequently, by inverting the CPMGs and recording the integral of each $\mathrm{T}_{2}^{\prime}$ component vs. $\tau$, the diffusion dynamics of each RTIL signal component is imprinted on the Hahn SEDs across the $\mathrm{T}_{2}^{\prime}$ distribution. This is excellently seen in Figs. 2a and 2c, which show the contour plots of the Hahn SED across the $\mathrm{T}_{2}^{\prime}$ distribution for the MCM-41 and SBA-15, respectively. The green lines are the projections of the integrated signal intensity onto the $\mathrm{T}_{2}^{\prime}$ axis. In both systems, two signal components are observed corresponding to the adsorbed and mobile RTIL cations. A third small peak at $\mathrm{T}_{2}^{\prime}<100 \mu$ s belongs apparently to silica-OH groups.

Figs. $2 \mathrm{~b}$ and $2 \mathrm{~d}$ show the relevant Hahn SEDs. Component 2 is observed to decay exponentially (blue line), as expected for immobilized (adsorbed) ions with $\mathrm{T}_{2}^{\prime} \sim 0.7 \mathrm{~ms}$. On the contrary, "mobile" $[\mathrm{BMIM}]^{+}$cations inside SBA-15 follow a nearly $\tau^{3}$ exponential decay (red line), while in the case of MCM-41 the data are best fitted with equation (2) and $\kappa=0.5$, indicating anomalous diffusion, close to SFD (orange color line). The apparent deviation from the theoretical SFD behavior is due to the biased nature of the random motion under the influence of Coulomb interactions. 

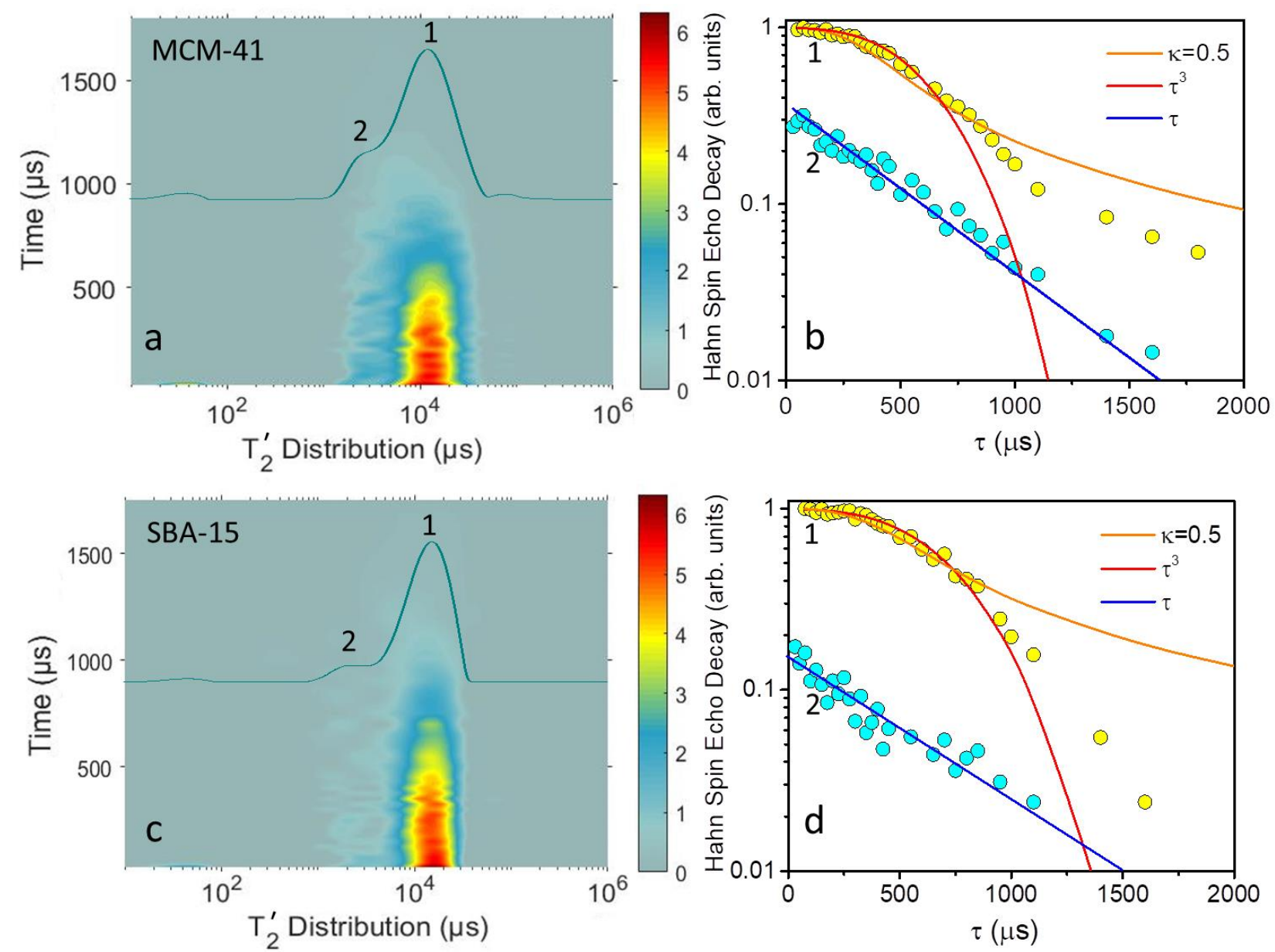

Figure $2 \mid{ }^{1} \mathrm{H}$ NMR $\mathrm{T}_{2}^{\prime}$-resolved Hahn SEDs of [BMIM] ${ }^{+}[\mathrm{TCM}]^{-}$confined in MCM-41 and

SBA-15 at RT. a, Hahn SEDs across the ${ }^{1} \mathrm{H}$ NMR $\mathrm{T}_{2}^{\prime}$ distribution of the RTIL confined in MCM-41. The dark cyan line is the projection of the signal intensity on the $\mathrm{T}_{2}^{\prime}$-axis. $\mathbf{b}$, The Hahn SED vs. $\tau$ of signals 1 and 2 in Fig. 2a. Signal 1 recalls SFD (orange color line), whereas signal 2 corresponds to adsorbed [BMIM] $]^{+}$cations (blue line - exponential decay). c, Hahn SEDs across the ${ }^{1} \mathrm{H}$ NMR $\mathrm{T}_{2}^{\prime}$ distribution of the RTIL confined in SBA-15. The dark cyan line is the projection of the signal intensity on the $\mathrm{T}_{2}^{\prime}$-axis. As previously, the two signals correspond to adsorbed and mobile [BMIM] $]^{+}$cations. d, The Hahn SED vs. $\tau$ of signals 1 and 2 in Fig. 2c. The diffusion of signal 1 is closer to unrestricted diffusion (red line).

To further validate these results, experiments were performed on two ionogels, with 
MCM-41, and SBA-15 soaked into $[\mathrm{BMIM}]^{+}[\mathrm{TCM}]^{-}$at concentrations $10 \%$ and $3 \%$ per weight, respectively. Figs. 3a and 3c show the relevant contour plots of the Hahn SEDs vs. the $\mathrm{T}_{2}^{\prime}$ distribution. In the case of MCM-41, three signal components are resolved corresponding to bulk $[\mathrm{BMIM}]^{+}$(signal 1), $[\mathrm{BMIM}]^{+}$inside the pores (signal 2), and $[\mathrm{BMIM}]^{+}$adsorbed on the silica walls (signal 3); the latter two components acquire precisely the same $\mathrm{T}_{2}^{\prime}$ values as those in Fig. 2a. Notably, the Hahn SED of signal 1 follows the exponential $\tau^{3}$ decay described by equation (1) (black line), while signal 2 decays according to the SFD mechanism (orange color line) and signal 3 decays exponentially (blue line), as expected for adsorbed [BMIM] ${ }^{+}$cations. This is the first time that the NMR signals of a multiphasic ionogel are resolved according to the diffusion characteristics of each component.
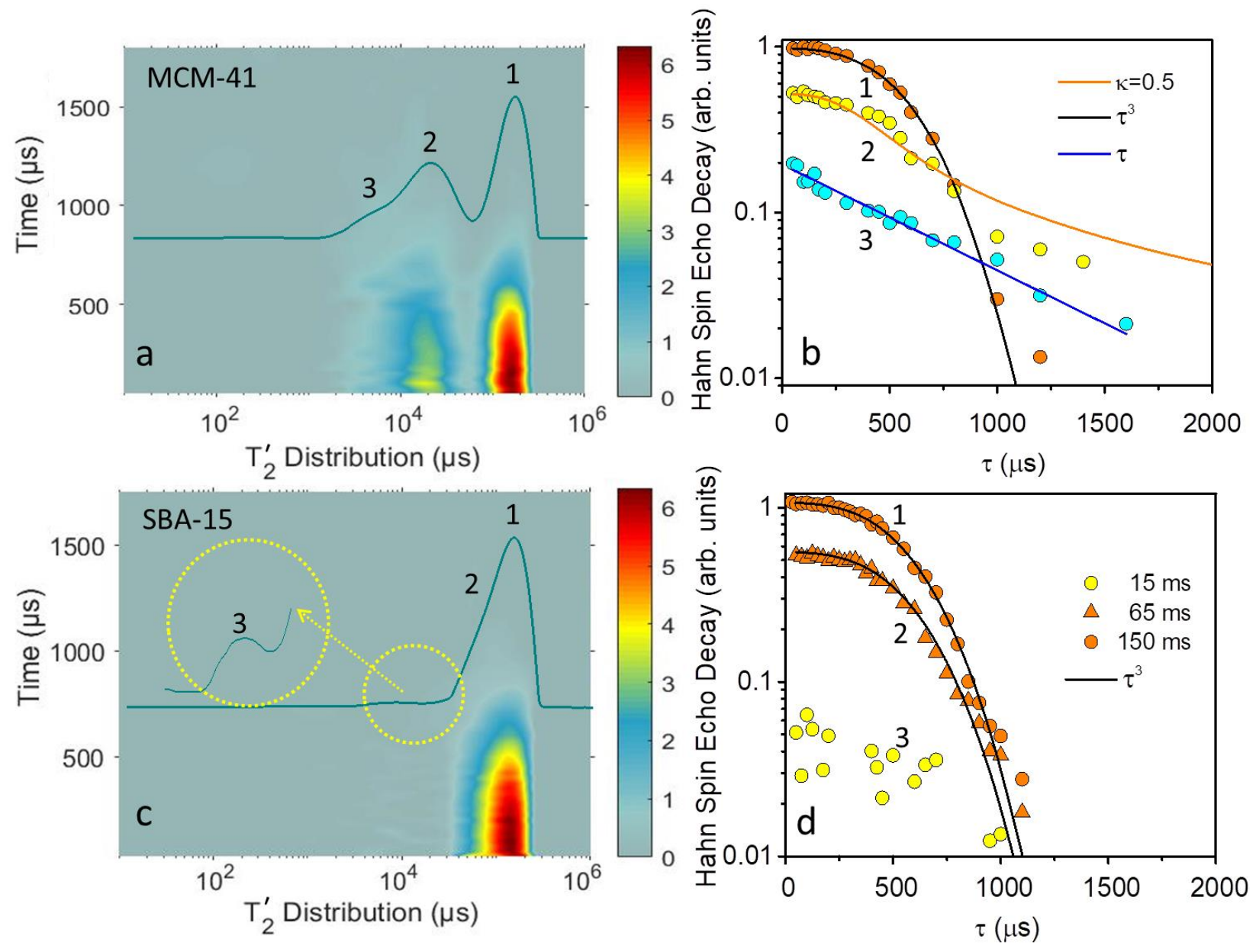
Figure $\left.3\right|^{1} \mathrm{H}$ NMR $\mathrm{T}_{2}^{\prime}$-resolved Hahn SEDs of oversaturated [BMIM] $]^{+}[\mathrm{TCM}]^{-}$/ MCM-41 and SBA-15 ionogels at RT. a, Hahn SEDs across the ${ }^{1} \mathrm{H}$ NMR $\mathrm{T}_{2}^{\prime}$ distribution of the MCM-41 ionogel. The dark cyan line is the projection of the signal intensity on the $\mathrm{T}_{2}^{\prime}$-axis. Three signals are observed corresponding to external bulk (signal 1), confined mobile (signal 2), and adsorbed (signal 3) $[\mathrm{BMIM}]^{+}$cations. b, The Hahn SED vs. $\tau$ of signals 1, 2, and 3 in Fig. 3a. As in Fig. 2, signal 1 follows unrestricted diffusion (black line), signal 2 exhibits SFD (orange color line), while signal 3 is decaying exponentially (blue line), as expected for immobilized cations. c, ${ }^{1} \mathrm{H}$ NMR Hahn SED across the $T_{2}^{\prime}$ distribution of the SBA-15 ionogel. The dark cyan line is the projection of the signal intensity on the $\mathrm{T}_{2}^{\prime}$-axis. Signals 1 and 2 belong to the external bulk $[\mathrm{BMIM}]^{+}$cations, whereas only a very weak signal of $[\mathrm{BMIM}]^{+}$entrapped into SBA-15 is observed, due to the high dilution. d, The Hahn SED of signals 1 and 2 follow unrestricted diffusion (black lines).

In the case of oversaturated SBA-15, the bulk out-of-pores NMR signal component dominates, as it corresponds to the main ionogel component. Notably the $\mathrm{T}_{2}^{\prime}$ distribution is slightly broadened, becoming asymmetric in the lower $\mathrm{T}_{2}^{\prime}$-values, whereas the relevant selfdiffusion coefficients D acquired by fitting with equation (1) are in the range $5.6-6.0 \cdot 10^{-11}$ $\mathrm{m}^{2} / \mathrm{s}$. The asymmetry might stem from a fast exchange between bulk external $[\mathrm{BMIM}]^{+}$cations and cations adsorbed on shallow nanopores on the external surfaces of SBA-15, as also implied by the nitrogen porosimetry in Fig. 1a. Most spectacularly, by magnifying the $\mathrm{T}_{2}^{\prime}$ region between 6-30 ms, the weak $\mathrm{T}_{2}^{\prime}$ signal of $[\mathrm{BMIM}]^{+}$inside the SBA-15 is unveiled. However, the relevant Hahn SED (signal 3 in Fig. 3d) is very weak and difficult to fit.

Fig. 4 shows ${ }^{1} \mathrm{H}$ NMR Hahn SED and 2D diffusion-relaxation (D $-\mathrm{T}_{2}^{\prime}$ ) experiments at 400K of the MCM-41 sample analyzed in Figs. 1, 2. At this temperature the RTIL is no more 
adsorbed on the pore walls, as already shown in Fig. 1d. However, the Hahn SED follows neither an exponential $\tau^{3}$ decay (i.e. according to equation (1)), nor a SFD according to equation (2), as clearly seen in Fig. 4a. Notably, an excellent fit is acquired by inverting the Hahn SED data according to equation (1) with a 1D Tikhonov regularization inversion algorithm ${ }^{14,15,27,28}$, corresponding to a doubly peaked distribution of the $D$ values with peaks at $\sim 3 \times 10^{-11} \mathrm{~m}^{2} / \mathrm{s}$ and $2 \times 10^{-10} \mathrm{~m}^{2} / \mathrm{s}$, respectively.
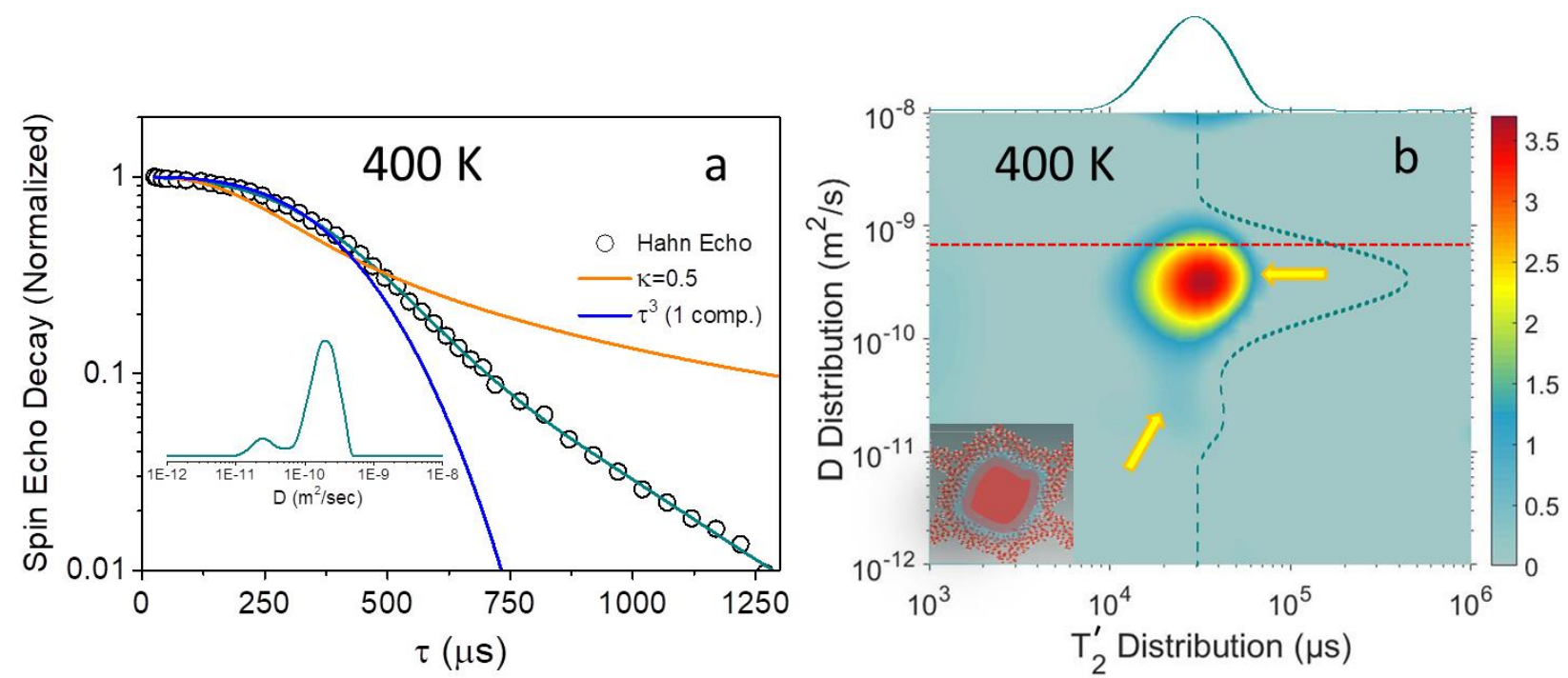

Figure $4 \mid 2 \mathrm{D}{ }^{1} \mathrm{H}$ NMR diffusion-relaxation $\left(\mathrm{D}-\mathrm{T}_{2}^{\prime}\right)$ spectrum at $400 \mathrm{~K}$. a, The ${ }^{1} \mathrm{H} \mathrm{NMR}$ Hahn SED of $[\mathrm{BMIM}]^{+}[\mathrm{TCM}]^{-}$confined in MCM-41 at $400 \mathrm{~K}$. The green line is the fit, and the inset the relevant self-diffusion coefficient D distribution, acquired by using a Tikhonof regularization inversion algorithm ${ }^{14,15}$, as explained in the text. The blue and orange color lines are the single exponential $\tau^{3}$ decay according to equation (1) and SFD, according to equation (2) with $\kappa=0.5$, respectively. b, The ${ }^{1} \mathrm{H}$ NMR D $-\mathrm{T}_{2}^{\prime}$ spectrum at $400 \mathrm{~K}$. At elevated temperatures adsorbed $[\mathrm{BMIM}]^{+}[\mathrm{TCM}]^{-}$molecules are detached from the MCM-41pore walls, however a distribution of $\mathrm{D}$ values is observed, with the slower D-component belonging to molecules close to the pore walls, due to motion impedance from hydrogen bonding. The red dotted line declares the D value of the bulk RTIL at 400K. The inset is schematic representation of the diffusion field 
inside a MCM-41 silica pore, with the slow diffusion close to the walls in light blue color.

To validate this result a $2 \mathrm{D}{ }^{1} \mathrm{H}$ NMR $\mathrm{D}-\mathrm{T}_{2}^{\prime}$ experiment was performed at $400 \mathrm{~K}$. In a $\mathrm{D}-\mathrm{T}_{2}^{\prime}$ experiment a sequence of CPMG SEDs is acquired with the pulse sequence described in Supplementary Fig. 4, and subsequently inverted with a 2D inversion algorithm ${ }^{14,15,27,28}$, where a distribution of diffusion processes is considered following the $\tau^{3}$ exponential decay described in equation (1). The relevant $\mathrm{D}-\mathrm{T}_{2}^{\prime}$ contour plot at $400 \mathrm{~K}$ is shown in Fig. 4b. A doubly peaked Ddistribution is observed in agreement with the Hahn SED experiment; the slightly higher $D$ values in the case of the $\mathrm{D}-\mathrm{T}_{2}^{\prime}$ experiment are due to the small sample heating by the long pulse-trains in the CPMG experiments. Furthermore, the low-D signal component acquires a slightly lower $\mathrm{T}_{2}^{\prime}$ value in comparison to the strong high-D signal; nevertheless, the two signals indicated with arrows in Fig. $4 \mathrm{~b}$ cannot be resolved in the $\mathrm{T}_{2}^{\prime}$ projection. The above experimental results witness the appearance of an inhomogeneous axial diffusion, with the slow diffusing RTIL component near the pore-walls, as schematically shown in the inset of Fig. 4b. Such low D-values are due to the impedance from hydrogen bond interactions between the RTIL and the silica silanol groups.

To comprehend the theoretical background of the above experimental results, free cluster static and molecular dynamic DFT calculations were performed with the ORCA software package ${ }^{29,30}$. Geometry optimizations showed that adsorption of the RTIL is governed by hydrogen bonding with the silanol groups of the silica surface. The non-polar butyl-"tail" of $[\mathrm{BMIM}]^{+}$appears to be repulsed from the polar surface of MCM-41, aligning almost vertically to the pore walls, as shown in Fig. 1a, and Supplementary Fig. 5. Furthermore, interaction energies and enthalpies showed that it is favorable for the IL to detach as ion pairs from the silica surface (Supplementary Table 2); this motion in pairs of ions restricts further free-tumbling in the limited pore space, as schematically shown in the upper right panel of Fig. 1a, thus favoring SFD. 
Notably, with increasing temperature, adsorbed $[\mathrm{BMIM}]^{+}$cations gradually detach, and for $\mathrm{T}>$ $360 \mathrm{~K}$ a single phase is observed as acquainted by the $\mathrm{T}_{2}^{\prime}$ distributions in Fig. $5 \mathrm{~b}$. Ab Initio Molecular Dynamics (AIMD) showed that for $\mathrm{T}=400 \mathrm{~K}$, $[\mathrm{BMIM}]^{+}[\mathrm{TCM}]^{-}$attains enhanced mobility because hydrogen bonds loosen and the ion pairs drift away from the surface, as seen in Figs. 5c-5e, and the Supplementary Video 1.

The SFD of $[\mathrm{BMIM}]^{+}[\mathrm{TCM}]^{-}$in MCM-41 might also explain the delayed freezing during cooling in comparison to SBA-15. The $\mathrm{T}_{2}^{\prime}$ dynamics can be mainly distinguished in two regions: the high-temperature region I and the low-temperature region II, separated by the glass transition temperature $\mathrm{T}_{\mathrm{g}}{ }^{31}$, as shown in Fig. 5a. In the case of MCM-41 glassification by cooling is evolving slower in the 1D single-file arrangement.
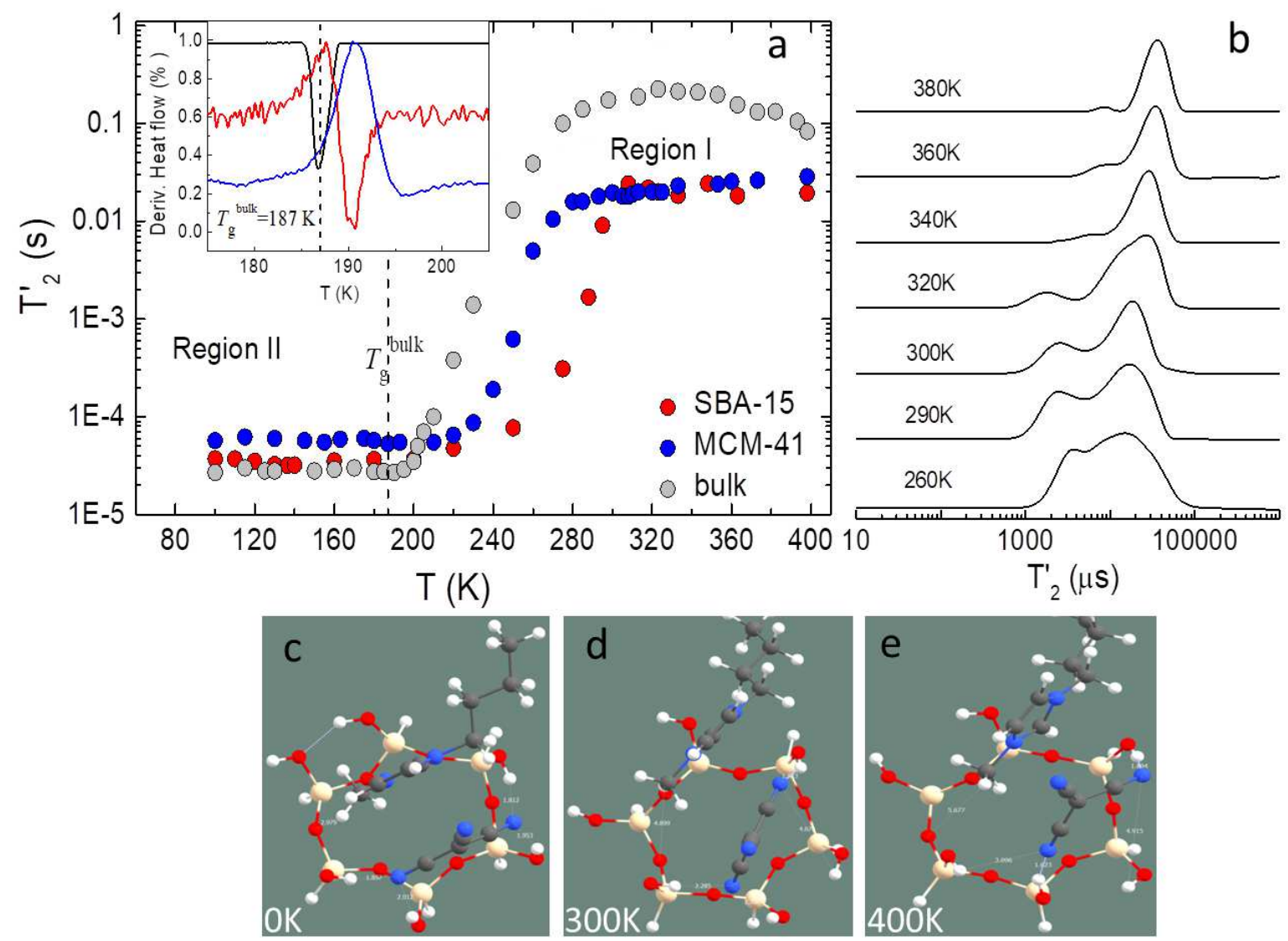

Figure $5 \mid{ }^{1} \mathrm{H}$ NMR $\mathrm{T}_{2}^{\prime}$ vs. T measurements combined with AIMD simulations. a, The mean 
$\mathrm{T}_{2}^{\prime} \quad$ vs. $\mathrm{T}$ for bulk and confined $[\mathrm{BMIM}]^{+}[\mathrm{TCM}]^{-}$in MCM-41 and SBA-15. The inset shows the derivatives of the heat flow in the relevant Differential Scanning Calorimetry (DSC) experiments. b, $\mathrm{T}_{2}^{\prime}$ distributions of $[\mathrm{BMIM}]^{+}[\mathrm{TCM}]^{-}$in $\mathrm{MCM}-41$ at various temperatures. c-e, AIMD simulations of $[\mathrm{BMIM}]^{+}[\mathrm{TCM}]^{-}$adsorbed on a MCM-41 silica fragment at $0 \mathrm{~K}, 300 \mathrm{~K}$ and 400K (instants from Supplementary Video).

In conclusion, by combining ${ }^{1} \mathrm{H}$ NMR diffusion experiments in different $\mathrm{T}_{2}^{\prime}$ relaxation windows with AIMD simulations, we have shown that the RTIL [BMIM] $]^{+}[\mathrm{TCM}]^{-}$when confined in the MCM-41 silica nanopores, exhibits an intriguing DMO effect. At room temperature, a layer of RTIL molecules is adsorbed on the pore-walls, with the tail of the $[\mathrm{BMIM}]^{+}$cations oriented almost vertically to the walls. However, at the center of the pores, free RTIL anion-cation pairs are constrained to diffuse collectively along the pore-axis, obeying a SFD mechanism. At elevated temperatures, the adsorbed RTIL molecules separate from the silica surface; however, existing hydrogen bond interactions hamper the motion of the RTIL molecules close to the walls, thus giving rise to a stratified axial diffusion. The convincing evidence for this extraordinary DMO effect provides key information regarding the use of RTILbased composites in energy storage applications. Furthermore, the applied NMR methodology is highly promising for enlightening complex multiphasic diffusion in a variety of fluidic processes ranging from living matter to nanofluidic technologies.

\section{Methods}

\section{$\underline{\text { Materials }}$}

The ionic liquid (IL) 1-butyl-3-methylimidazolium tricyanomethanide $[\mathrm{BMIM}]^{+}[\mathrm{TCM}]^{-}$(mass fraction purity $>98 \%$, with $720 \mathrm{ppm} \mathrm{H}_{2} \mathrm{O}$ content) was purchased by IoLiTec GmbH. Mesoporous materials MCM-41 (pore size $4.09 \mathrm{~nm}$ ) and SBA-15 (pore size of $7.31 \mathrm{~nm}$ ) were acquired by Sigma Aldrich Corp. and ACS Material LLC, respectively. 
Samples for the NMR experiments were prepared inside $\Gamma$-shaped NMR quartz tubes having inner/outer diameters of $3.0 \mathrm{~mm} / 5.0 \mathrm{~mm}$ respectively, and lengths of $25 \mathrm{~mm}$. Three types of samples were prepared: bulk $[\mathrm{BMIM}]^{+}[\mathrm{TCM}]^{-}$and two ionogels $[\mathrm{BMIM}]^{+}[\mathrm{TCM}]^{-} / \mathrm{MCM}-41$, $[\mathrm{BMIM}]^{+}[\mathrm{TCM}]^{-} / \mathrm{SBA}-15$ fabricated by intruding the IL into MCM-41 and SBA-15, respectively. Two samples were prepared for each category, for data reproducibility purposes.

The sample preparation involved a specially constructed gas manifold system connected to a turbomolecular pump. The quartz tube, filled with about $20 \mathrm{mg}$ of MCM-41 or SBA-15, was attached to the manifold, and each sample was outgassed at a high vacuum $\left(10^{-7} \mathrm{mbar}\right)$ and $180^{\circ} \mathrm{C}$ for 24 hours. Subsequently, after bringing the sample to ambient temperature, a toggle valve connecting the manifold to the turbomolecular pump was closed, and a second valve was opened to suction a methanol/IL solution and soak the sample under vacuum. The concentration of the IL in the solution was determined to have the IL phase confined into the pores of the sample and avoid excess IL in the quartz tube after methanol evaporation. To calculate the IL concentration that suffices to fill the pores, the total pore volume, the mass of the sample, and the free volume of the quartz tube were considered. Methanol was slowly evaporated at $50^{\circ} \mathrm{C}$ at ambient pressure and then the samples further outgassed at $90^{\circ} \mathrm{C}$ under high vacuum for 24 hours before been flame sealed. The bulk IL sample was placed into a quartz tube with a syringe, further outgassed at $90^{\circ} \mathrm{C}$ under a high vacuum for 24 hours, and flame sealed. As described below, porosimetry results before and after IL impregnation (shown in Supplementary Fig. 1) confirmed the complete blockage of the MCM and SBA pores by the IL.

\section{NMR experiments}

${ }^{1} \mathrm{H}$ NMR experiments were performed in the stray field of a $4.7 \mathrm{~T}$ Bruker superconducting magnet providing a $34.7 \mathrm{~T} / \mathrm{m}$ constant magnetic field gradient at ${ }^{1} \mathrm{H}$ NMR Larmor frequency 101.168 MHz. 
A broadband coherent pulse NMR spectrometer was used, operating in the frequency range 5 $\mathrm{MHz}-1 \mathrm{GHz}$. The $\pi / 2$ pulse length was set to $2 \mu \mathrm{s}$; with this setup, a slice of the sample with a thickness of $\sim 0.7 \mathrm{~mm}$ along the $\mathrm{z}$-axis was possible to excite. The ${ }^{1} \mathrm{H}$ NMR effective spin-spin relaxation $\left(\mathrm{T}_{2}^{\prime}\right)$ and self-diffusion coefficient $\mathrm{D}$ measurements were accomplished by combining the Hahn echo and the Carr-Purcell-Meiboom-Gill (CPMG) pulse sequences, as described in the Supplementary Fig. 4 and the relevant text.

For the $\mathrm{T}_{2}^{\prime}$ vs. temperature measurements, in the temperature range $100 \mathrm{~K}$ to $400 \mathrm{~K}$, an Oxford $1200 \mathrm{CF}$ continuous-flow cryostat was employed, with an accuracy of $\pm 0.1 \mathrm{~K}$. The samples were initially cooled down to $100 \mathrm{~K}$, and the NMR measurements were obtained on heating to rule out any thermal hysteresis effects.

\section{$\underline{\text { Computational Methods }}$}

In order to qualitatively examine the Silica - RTIL system and validate the experimental results, a series of free cluster DFT simulations were conducted. Geometry optimizations and frequency analysis were performed using the ORCA suite of programs and the PBEh-3c parametrized functional $^{29,30,32}$. For the simulation of the silica surface, a ring of six silanol tetrahedra was cropped from the cif file in ref. $^{33}$. The cif file was also used to simulate the MCM-41 pore structure in Fig. 1a and inset in Fig. 4b. Molecular structures processing was conducted with Chemcraft software ${ }^{34}$.

The desorption of the IL from the silica surface was examined with the help of Ab Initio Molecular Dynamics (AIMD) simulations, using the ORCA suite of programs. The PBEh-3c parametrized functional was implemented, along with the Beredsen thermostat, and the time step was fixed at $0.5 \mathrm{fs}^{32}$. Geometry constraints were applied on silicon atoms in order to simulate the solid surface of the silica better. 


\section{Acknowledgments:}

J.H., A.C., and G.P., acknowledge support by the Khalifa University of Science and Technology under Award No. CIRA-2020-051. L.G., M.K., and M.F. acknowledge support by the project MIS 5047810, through the Operational Program «Human Resources Development, Education and Lifelong Learning 2014-2020», co-financed by Greece and the European Union (European Social Fund- ESF).

\section{Author contributions:}

Conceptualization: GP, JH, MF. Methodology: GP, JH, MF, GR, SA. Investigation: MK, LG, NT, SO, NP, AC, GR. Funding acquisition: JH, GP, MF. Project administration: GP, JH, MF. Supervision: GP, JH, MF, GR, SA. Writing - review \& editing: GP, MF, JH, MK, NT, SA, GR.

Competing Interests statement: Authors declare that they have no competing interests.

\section{Additional Information}

Supplementary Information is included in the SI document. This includes, Supplementary Text, Supplementary Figs. S1 to S6, Supplementary Tables S1 to S4, and Supplementary Video S1.

\section{References}

1. Berezhkovskii, A. \& Hummer, G. Single-File Transport of Water Molecules through a Carbon Nanotube. Phys. Rev. Lett. 89, 064503 (2002).

2. Ghoufi, A., Szymczyk, A. \& Malfreyt, P. Ultrafast diffusion of Ionic Liquids Confined in Carbon Nanotubes. Sci Rep 6, 28518 (2016).

3. Chaban, V. V. \& Prezhdo, O. V. Nanoscale Carbon Greatly Enhances Mobility of a Highly Viscous Ionic Liquid. ACS Nano 8, 8190-8197 (2014).

4. Chathoth, S. M. et al. Fast diffusion in a room temperature ionic liquid confined in mesoporous carbon. EPL 97, 66004 (2012). 
5. Iacob, C. et al. Enhanced charge transport in nano-confined ionic liquids. Soft Matter 8, 289-293 (2012).

6. Garaga, M. N. et al. A long-chain protic ionic liquid inside silica nanopores: enhanced proton mobility due to efficient self-assembly and decoupled proton transport. Nanoscale 10, 1233712348 (2018).

7. Wang, Y.-L. et al. Microstructural and Dynamical Heterogeneities in Ionic Liquids. Chem. Rev. 120, 5798-5877 (2020).

8. Broussely, M. et al. Main aging mechanisms in Li ion batteries. Journal of Power Sources 146, 90-96 (2005).

9. Balducci, A., Bardi, U., Caporali, S., Mastragostino, M. \& Soavi, F. Ionic liquids for hybrid supercapacitors. Electrochemistry Communications 6, 566-570 (2004).

10. $\mathrm{Xu}, \mathrm{W}$. Solvent-Free Electrolytes with Aqueous Solution-Like Conductivities. Science 302, 422-425 (2003).

11. Gupta, A. K., Verma, Y. L., Singh, R. K. \& Chandra, S. Studies on an Ionic Liquid Confined in Silica Nanopores: Change in $T_{\mathrm{g}}$ and Evidence of Organic-Inorganic Linkage at the Pore Wall Surface. J. Phys. Chem. C 118, 1530-1539 (2014).

12. Stejskal, E. O., \& Tanner, J. E. Spin Diffusion Measurements: Spin Echoes in the Presence of a Time-Dependent Field Gradient. The Journal of Chemical Physics 42, 288-292 (1965).

13. Kimmich, R., Unrath, W., Schnur, G. \& Rommel, E. NMR measurement of small selfdiffusion coefficients in the fringe field of superconducting magnets. Journal of Magnetic Resonance (1969) 91, 136-140 (1991).

14. Hassan, J., Diamantopoulos, G., Gkoura, L., et al. Ultrafast Stratified Diffusion of Water Inside Carbon Nanotubes; Direct Experimental Evidence with 2D D-T2 NMR Spectroscopy. J. Phys. Chem. C 122, 10600-10606 (2018). 
15. Gkoura, L., Diamantopoulos, G., Fardis, M., et al. The peculiar size and temperature dependence of water diffusion in carbon nanotubes studied with 2D NMR diffusion-relaxation D- T $_{2 \text { eff }}$ spectroscopy. Biomicrofluidics 14, 034114 (2020).

16. Das, A. et al. Single-File Diffusion of Confined Water Inside SWNTs: An NMR Study. ACS Nano 4, 1687-1695 (2010).

17. Lynch, C. I., Rao, S. \& Sansom, M. S. P. Water in Nanopores and Biological Channels: A Molecular Simulation Perspective. Chem. Rev. 120, 10298-10335 (2020).

18. Kondrashova, D. et al. Scale-dependent diffusion anisotropy in nanoporous silicon. Sci Rep 7, 40207 (2017).

19. Ylihautala, M., Jokisaari, J., Fischer, E. \& Kimmich, R. Fringe field NMR diffusometry of anomalous self-diffusion in molecular sieves. Phys. Rev. E 57, 6844-6850 (1998).

20. Kärger, J., Pfeifer, K., Vojta, G. Time correlation during anomalous diffusion in fractal systems and signal attenuation in NMR field-gradient spectroscopy. Phys. Rev. A 37, 4514-4517 (1988).

21. Mikheev, V. A., Maidanov, V. A. \& Mikhin, N. P. Quasi-one-dimensional diffusion of He3 impurities in molecular solid hydrogen. Physica B+C 107, 275-276 (1981).

22. Wei, Q. Single-File Diffusion of Colloids in One-Dimensional Channels. Science 287, 625627 (2000).

23. Lutz, C., Kollmann, M. \& Bechinger, C. Single-file diffusion of colloids in one-dimensional channels. Physical Review Letters 93, 026001 (2004).

24. Hahn, K., Kärger, J. \& Kukla, V. Single-File Diffusion Observation. Phys. Rev. Lett. 76, 2762-2765 (1996).

25. Dutta, A. R. et al. Relationship between single-file diffusion of mixed and pure gases in dipeptide nanochannels by high field diffusion NMR. Chem. Commun. 51, 13346-13349 (2015). 
26. Korb, J.-P. Nuclear magnetic relaxation of liquids in porous media. New J. Phys. 13, 035016 (2011).

27. Mitchell, J., Chandrasekera, T. C., Gladden, L. F. Numerical estimation of relaxation and diffusion distributions in two dimensions. Prog. Nucl. Magn. Reson. Spectroscopy 62, 34-50 (2012).

28. Day, J. On the inversion of diffusion NMR data: Tikhonov regularization and optimal choice of the regularization parameter. J. Magn. Reson. 211, 178-185 (2011).

29. Neese, F. The ORCA program system. Wiley Interdisciplinary Reviews: Computational Molecular Science 2(1), $73-78$ (2012).

30. Neese, F. Software update: the ORCA program system, version 4.0. Wiley interdisciplinary Reviews: Computational Molecular Science 8 (1), e1327 (2017).

31. Tripathi, A. K., Verma, Y. L. \& Singh, R. K. Thermal, electrical and structural studies on ionic liquid confined in ordered mesoporous MCM-41. J. Mater. Chem. A 3, 23809-23820 (2015).

32. Grimme, S., Brandenburg, J. G., Bannwarth, C., Hansen, A. J. Chem. Phys. 143, 054107 (2015).

33. Ugliengo, P., Sodupe, M., Musso, F., Bush, I. J., Orlando, R., Dovesi, R. Realistic models of hydroxylated amorphous silica surfaces and MCM-41 mesoporous material simulated by largescale periodic B3LYP calculations. Adv. Mater. 20, 1 (2008).

34. Chemcraft - graphical software for visualization of quantum chemistry computations. https://www.chemcraftprog.com. 


\section{Supplementary Files}

This is a list of supplementary files associated with this preprint. Click to download.

- SupplementaryInformation.pdf

- SupplementaryMovie.mp4 